\title{
Preparation of Carbamazepine-Nicotinamide Cocrystal
}

\author{
Huirong Ying1, Jinna Zhang2, Chengjun Jiang2* \\ ${ }^{1}$ Zhejiang Hisoar Pharmaceutical Co., Ltd., Taizhou, China \\ ${ }^{2}$ School of Biological and Chemical Engineering, Zhejiang University of Science and Technology, Hangzhou, China \\ Email: ${ }^{j}$ cj312@zust.edu.cn
}

How to cite this paper: Ying, H.R., Zhang, J.N. and Jiang, C.J. (2021) Preparation of Carbamazepine-Nicotinamide Cocrystal. Open Access Library Journal, 8: e7605. https://doi.org/10.4236/oalib.1107605

Received: June 2, 2021

Accepted: July 17, 2021

Published: July 20, 2021

Copyright (c) 2021 by author(s) and Open Access Library Inc.

This work is licensed under the Creative Commons Attribution International License (CC BY 4.0).

http://creativecommons.org/licenses/by/4.0/

\section{(c) (i) Open Access}

\begin{abstract}
The focus of this investigation was to prepare the cocrystal of carbamazepine (CBZ) using nicotinamide as a coformer and to compare its different preparation methods. The cocrystal was prepared by grinding, solvent-Assisted grinding and ultrasound-assisted cocrystallization methods. They were characterized by Fourier transform infrared spectroscopy (FT-IR), powder X-ray diffraction (PXRD), and morphology by electron microscopy. In conclusion, ultrasound can be applied as a process intensification parameter along with cooling or slurry cocrystallization to produce pure cocrystals under conditions that might result in crystal mixtures in conventional processes.
\end{abstract}

\section{Subject Areas}

Chemical Engineering \& Technology

\section{Keywords}

Carbamazepine, Nicotinamide, Cocrystal, Ultrasound

\section{Introduction}

Carbamazepine (CBZ) was first approved for the US market by the FDA in 1968 under the brand name Tegretol $^{\circledR}$ and currently approved as an anticonvulsant for selected epileptic seizures and the treatment of pain associated with trigeminal neuralgia [1]. CBZ is an iminostilbene derivative, which is structurally related to tricyclic antidepressants, with anticonvulsive and pain reduction due to a decreased potentiation of synaptic transmission in the affected areas [2]. CBZ is classified as a class 2 drug under the biopharmaceutics classification system [3]. Class 2 drug substances are characterized as having low solubility in an aqueous media, but a high permeability across the human intestinal membrane or an ap- 
propriately predictive in vitro model [4]. Therefore, formulation strategies are aimed at improving the solubility and dissolution rates in order to enhance the bioavailability of this highly permeable drug. The existence of a dihydrate and four anhydrous polymorphic forms of CBZ further complicates its formulation [5] [6]. CBZ polymorphs have been shown to yield different dissolution profiles and a significant difference in pharmacokinetic profiles when studied in dogs [7]. Nicotinamide (NCT) is a generally recognized as safe class 1 chemical and is often utilized in much larger doses to treat high cholesterol than seen in cocrystal formation [8] [9]. Nicotinamide has been widely used as a coformer [10] [11] [12] [13]. The preparation method of cocrystal has very important meaning in the preparation of cocrystal [14]. This article compares the different preparation methods of CBZ-NCT cocrystal, which is of great significance to the preparation of CBZ-NCT cocrystal.

\section{Materials and Methods}

\subsection{Materials}

Carbamazepine and nicotinamide were purchased from Energy Chemical (Shanghai, China). Solvents were purchased from Shanghai Lingfeng Chemical Reagent Co., Ltd. (Shanghai, China).

\subsection{Methods}

Cocrystal Synthesis

CBZ-NCT cocrystal was synthesized using grinding, solvent-Assisted grinding and ultrasound-assisted cocrystallization [15].

\subsection{Characterization of CBZ-NCT Cocrystal}

Fourier Transform Infrared Spectroscopy (FT-IR) spectra were recorded on a Perkin-Elmer Spectrum BX FTIR system, equipped with a deuterium triglycine sulfate detector. The scanrange was $500-4000 \mathrm{~cm}^{-1}$, using eight scans per spectrum with a resolution of $1 \mathrm{~cm}^{-1}$. Spectra were obtained in the transmission mode in $\mathrm{KBr}$ pellets.

Powder X-ray diffraction (PXRD) patterns were collected using Rigaku X-ray polycrystalline diffractometer. Samples were placed onto a silicon wafer slide. Generator settings were $40 \mathrm{kV}$ with a current of $40 \mathrm{~mA}$ used for the measurement. Data were collected $(n=3)$ in the range $2 \theta=5^{\circ}$ to $40^{\circ}$ at a scanning rate of $5 \%$ min with a scan rate of $0.04^{\circ} /$ time and a slit width of $6.0 \mathrm{~mm}$.

Microscopy was performed using Bai Datong wireless connection high-definition digital wifi microscope.

\section{Results}

\subsection{Fourier Transform Infrared Spectroscopy}

Also known as dry grinding or solid state grinding, mechanochemical grinding was the more typically used method of cocrystal synthesis. The FT-IR spectra of 
commercial CBZ and CBZ USP grade, recorded in $\mathrm{KBr}$ pellets. Figure 1 shows the FT-IR spectrum of CBZ, NCT, their physical mixture, and cocrystal prepared by solvent-free grinding.

It can be seen from Figure 1 that through simple solvent-free grinding, CBZ and NCT cannot form a cocrystal. One huge drawback with mechanochemical grinding in the solid state is that, with no heating stage involved in the process, there are numerous cases where the energy required to complete the cocrystallization of compounds is lacking. One method to overcome this is the induction of a small amount of water or a solvent to the ball milling mechanism, which acts as a catalyst assisting the process in a process known as Liquid-assisted grinding (LAG). LAG (also known as solvent-drop grinding or wet granulation) is mediated by a liquid phase.

Then we use solvent-assisted grinding, and the result is shown in Figure 2. Figure 2 shows the FT-IR spectrum of CBZ, NCT, their physical mixture, and cocrystal prepared by grinding with solvent. Dichloromethane (DCM), acetone (CP), n-butyl alcohol (NBA), n-propyl alcohol (NPA), Acetonitrile (ACN), ethyl alcohol (EA).

It can be seen from Figure 2 that the method of adding liquid to assist grinding cannot be CBZ and NTC to form a cocrystal.

Figure 3 shows the FTIR spectrum of CBZ, NCT, their physical mixture, and cocrystal prepared by ultrasound-assisted cocrystallization.

Figure 3 shows the FTIR spectrum of CBZ, NCT, and ultrasound-assisted cocrystallization prepared by different solvents. The stretching of the primary amide group in CBZ was represented by absorption bands at 3464 and 3163 $\mathrm{cm}^{-1}$, respectively, which could be assigned to the free anti-NH (asymmetrical) and hydrogen-bonded syn-NH (symmetrical) vibration. The absorption band at $1679 \mathrm{~cm}^{-1}$ was due to carbonyl $(-\mathrm{C}=\mathrm{O})$ stretching. It indicated that when form cocrystal, absorption bands at 3446,3391 and $3210 \mathrm{~cm}^{-1}$. Absorption bands due to carbonyl $(-\mathrm{C}=\mathrm{O})$ andvibrations appeared at 1683 and $1660 \mathrm{~cm}^{-1}$, respectively.

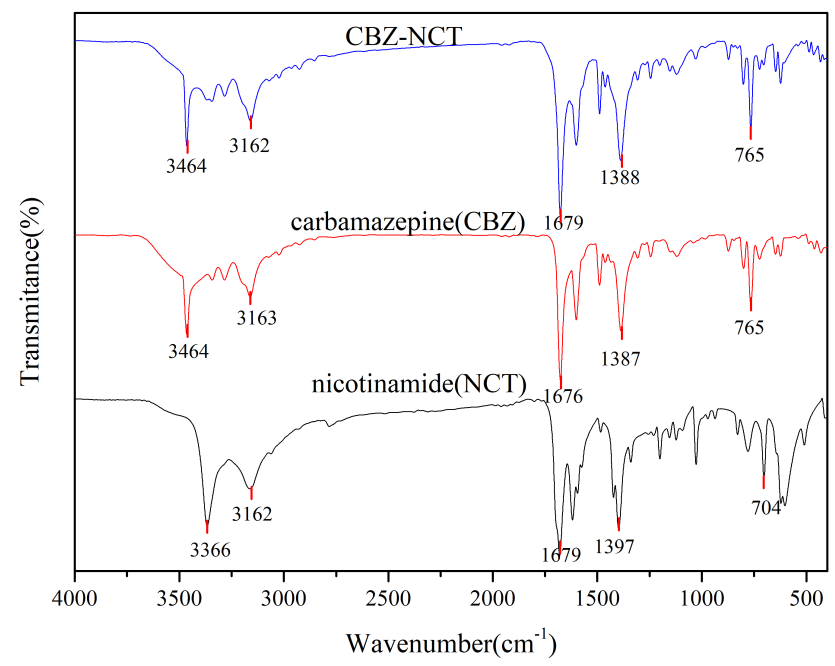

Figure 1. FTIR spectra of CBZ, NCT, and grinding mixture. 


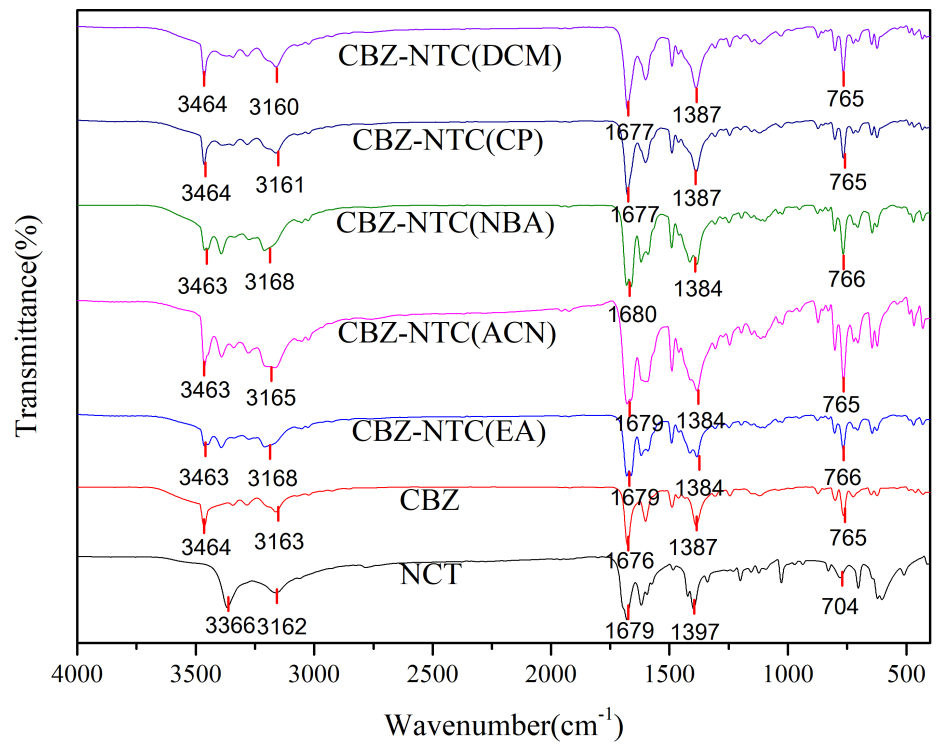

Figure 2. FTIR spectra of CBZ, NCT, and grinding with solvent.

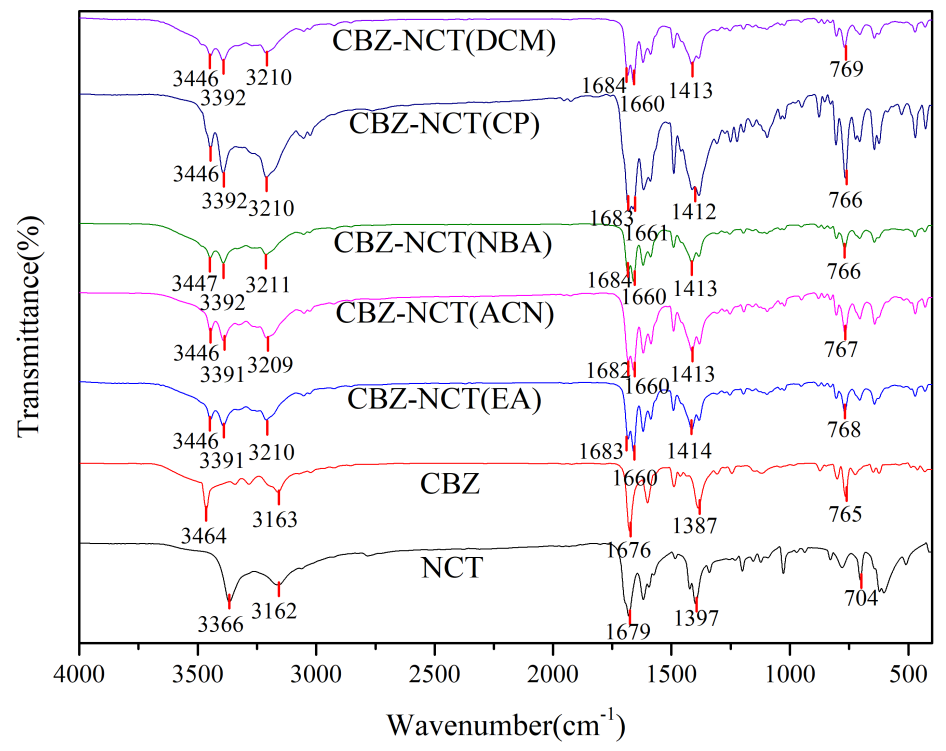

Figure 3. FTIR spectra of CBZ, NCT, and ultrasound induction product.

\subsection{Powder X-Ray Diffraction}

Figure 4 shows the PXRD spectrum of CBZ, NCT, their physical mixture prepared by solvent-free grinding.

It can be seen from Figure 4 that the grinding is only physical mixing.

Figure 5 shows the PXRD spectrum of CBZ, NCT, their physical mixture by grinding with solvent.

It can be seen from Figure 5 that the grinding by adding a solvent is only a physical mixing.

It can be seen from Figure 6 that through ultrasound induction, CBZ and NCT have formed cocrystal. The PXRD of CBZ forms showed sharp peaks at $2 \theta$ values of $13.0^{\circ}, 15.2^{\circ}, 15.8^{\circ}, 18.6^{\circ}, 19.4^{\circ}, 23.8^{\circ}, 26.68^{\circ}$, and $27.52^{\circ}$, respectively. 
Similarly, the diffractogram of NCT showed peaks at $2 \theta$ values of $14.72^{\circ}, 22.12^{\circ}$, $25.72^{\circ}, 27.24^{\circ}$, and $40.28^{\circ}$. Cocrystal showed sharp peaks at $6.68^{\circ}, 8.92^{\circ}, 10.2^{\circ}$, $13.32^{\circ}, 20.44^{\circ}, 23.04^{\circ}, 26.48^{\circ}, 28.12^{\circ}$.

\subsection{Electron Microscopy}

Solvent is the fundamental and foremost important factor for promoting or inhibiting certain crystal motif and to produce correlated effects to the success of required drug material compatibility for the end-user. The use of different solvents during crystallization profoundly affects the crystal habit of the purifified drug, leading to the variation in raw material characteristics such as flowability, compaction, chemical stability, dissolution and packing. Ultrasound-assisted cocrystallization in different solvent are shown in Figure 7.

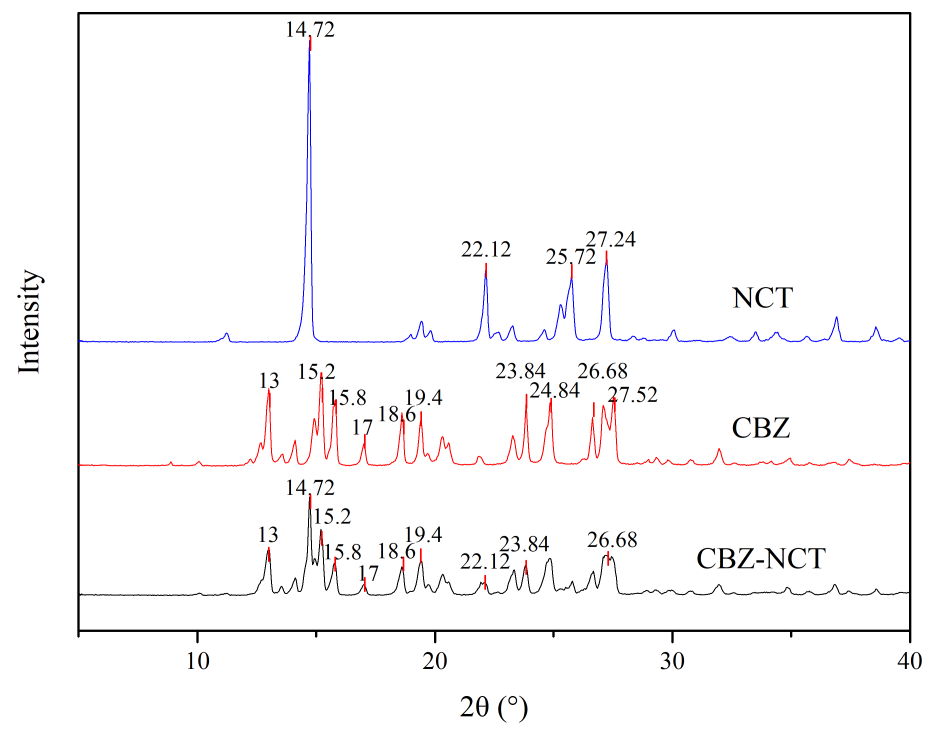

Figure 4. PXRD spectra of CBZ, NCT, and grinding mixture.

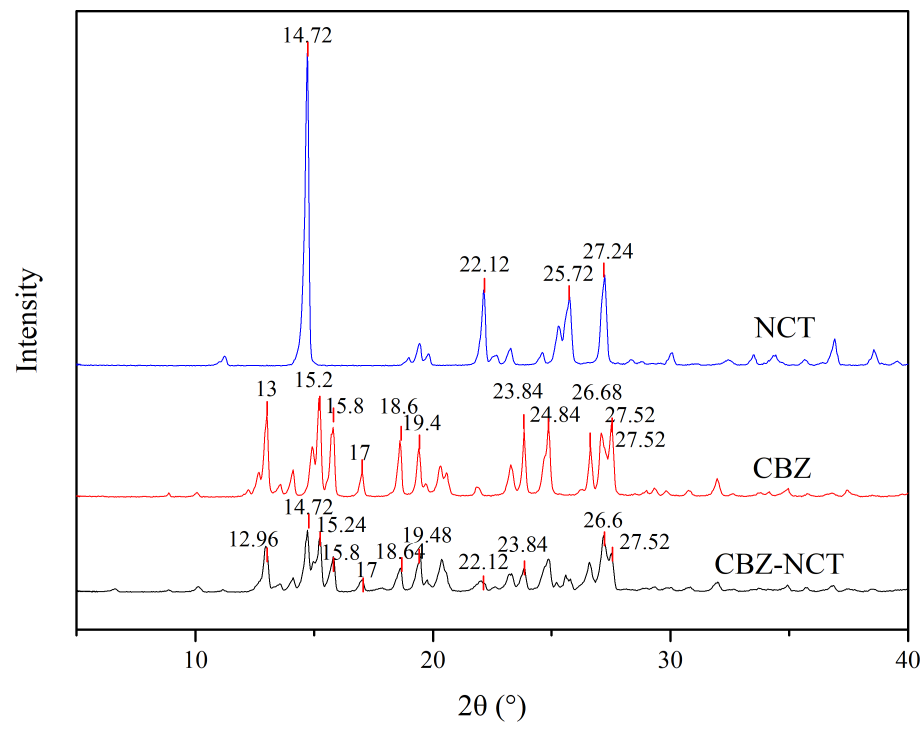

Figure 5. PXRD spectra of CBZ, NCT, and grinding mixture. 


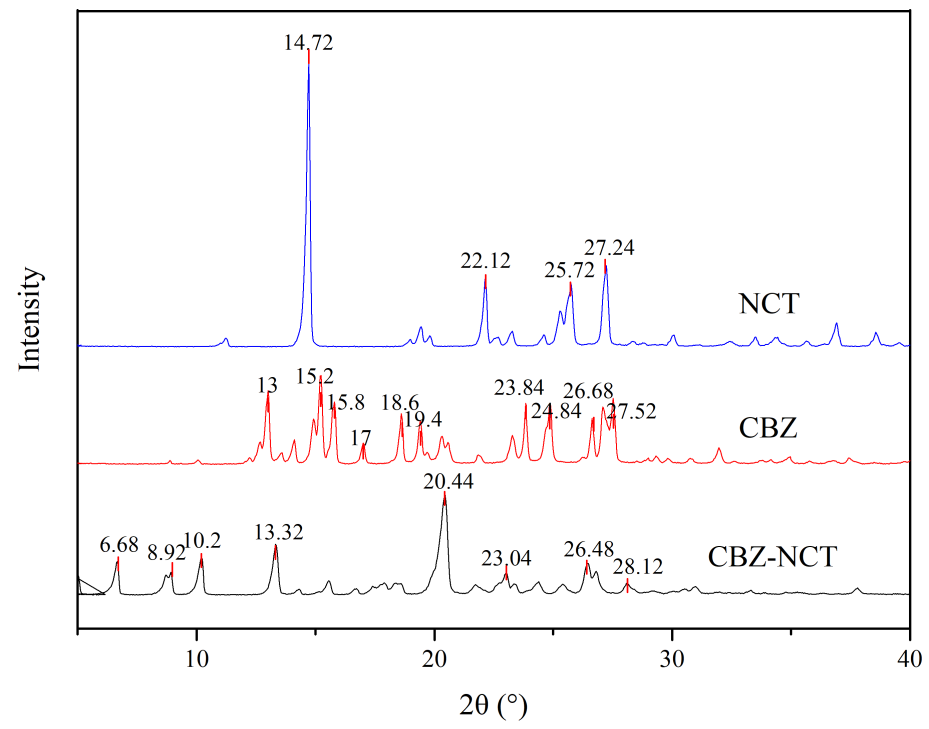

Figure 6. PXRD spectra of CBZ, NCT, and ultrasound induction product.

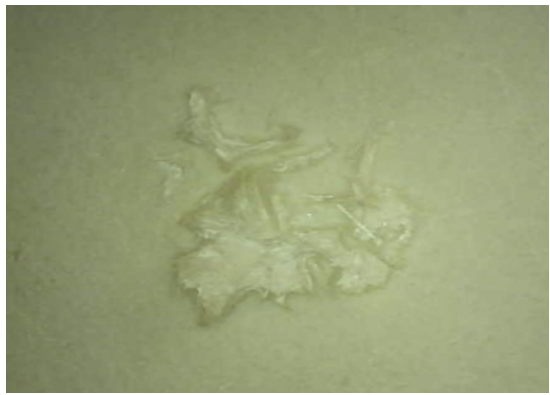

(a)

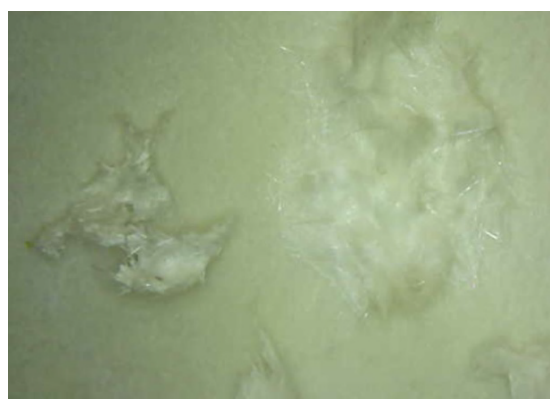

(c)

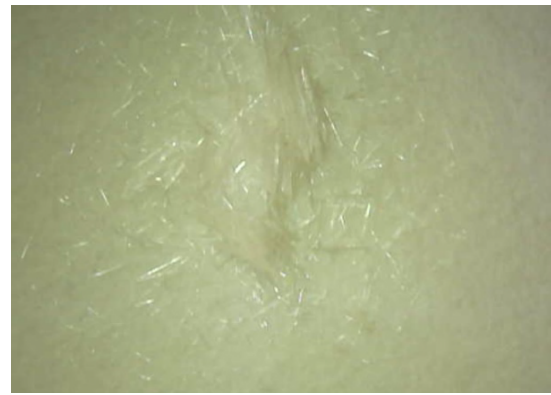

(b)

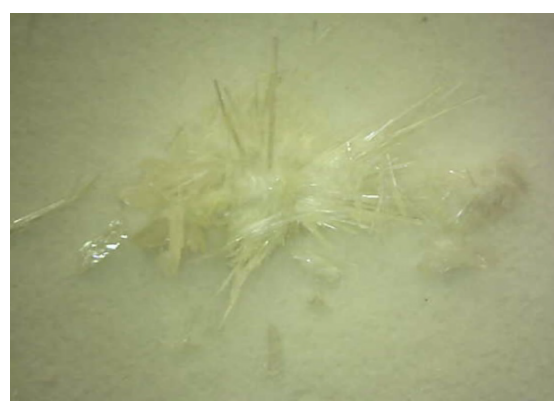

(d)

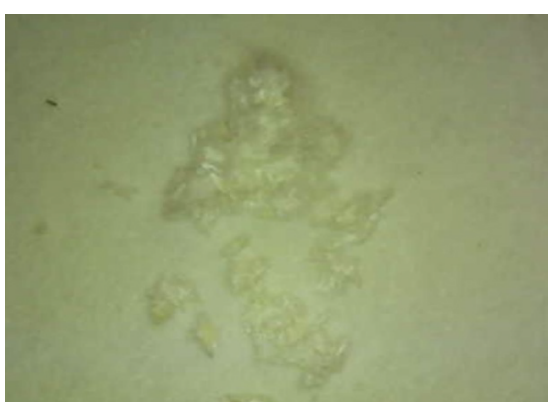

(e)

Figure 7. Crystal habit in different solvent. (a) CBZ-NCT (EA); (b) CBZ-NCT (ACN); (c) CBZ-NCT (NBA); (d) CBZ-NCT (CP); (e) CBZ-NCT (DCM). 
The effect of solvents on ultrasound-assisted cocrystallization in solution is discussed here. From the captured microscopic images, crystals in different solvents show different morphologies CBZ has polar functional groups as hydroxyl, amide and carbonyl groups and $\mathrm{CH}$ as the non-polar. Among the selected solvents, polar protic solvents such as isopropyl alcohol strongly allow them to dissolve both positively and negatively charged species to participate in intermolecular force via hydrogen bonding. Whereas the polar aprotic solvents such as acetone, ethylacetate and acetonitrile solvate only positive ions but not negative ions which results in the lack of hydrogen bonding. This result indicates that the type of solvent dramatically influences the shape of cocrystal with respect to the solubility of solute, solvent polarity, evaporation number of solvent and rate of generation of supersaturation in the solution.

\section{Conclusion}

With an increasing interest in cocrystals due to various advantages, demand for largescale cocrystallization techniques is rising. Ultrasound can be applied as a process intensification parameter along with cooling or slurry cocrystallization to produce pure cocrystals under conditions that might result in crystal mixtures in conventional processes. The current research demonstrated that the ultrasound-assisted cocrystallization can be an effective method in the production of CBZ-NCT cocrystals. There is hope that ultrasound-assisted cocrystallization will be realized in industry.

\section{Conflicts of Interest}

The authors declare no conflicts of interest.

\section{References}

[1] Post, R.M., Uhde, T.W., Roy-Byrne, P.P. and Joffe, R.T. (1986) Antidepressant Effects of Carbamazepine. The American Journal of Psychiatry, 143, 29-34. https://doi.org/10.1176/ajp.143.1.29

[2] Granger, P., Biton, B., Faure, C., Vige, X., Depoortere, H., Graham, D., et al. (2005) Modulation of the Gamma-Aminobutyric Acid Type A Receptor by the Antiepileptic Drugs Carbamazepine and Phenytoin. Molecular Pharmacology, 47, 1189-1196.

[3] Kasim, N.A., Whitehouse, M., Ramachandran, C., Bermejo, M., Lennernäs, H., Hussain, A.S., et al. (2003) Molecular Properties of WHO Essential Drugs and Provisional Biopharmaceutical Classification. Molecular Pharmacology, 1, 85-96. https://doi.org/10.1021/mp034006h

[4] Ku, M.S. (2008) Use of the Biopharmaceutical Classification System in Early Drug Development. The AAPS Journal, 10, 208-212. https://doi.org/10.1208/s12248-008-9020-0

[5] Grzesiak, A.L., Lang, M., Kim, K. and Matzger, A.J. (2003) Comparison of the Four Anhydrous Polymorphs of Carbamazepine and the Crystal Structure of form I. Journal of Pharmaceutical Sciences, 92, 2260-2271. https://doi.org/10.1002/jps.10455

[6] Rustichelli, C., Gamberini, G., Ferioli, V., Gamberinig, M.C., Ficarra, R. and Toma- 
sini, S. (2000) Solid-State Study of Polymorphic Drugs: Carbamazepine. Journal of Pharmaceutical and Biomedical Analysis, 23, 41-54. https://doi.org/10.1016/S0731-7085(00)00262-4

[7] Kobayashi, Y., Ito, S., Itai, S. and Yamamoto, K. (2000) Physicochemical Properties and Bioavailability of Carbamazepine Polymorphs and Dihydrate. International Journal of Pharmaceutics, 193, 137-146. https://doi.org/10.1016/S0378-5173(99)00315-4

[8] Das, B. and Baruah, J.B. (2011) Supramolecular Synthons and Hydrates in Stabilization of Multicomponent Crystals of Nicotinamide and Isonicotinamide with N-Containing Aromatic Dicarboxylic Acids. Crystal Growth \& Design, 11, 5522-5532. https://doi.org/10.1021/cg201096c

[9] Remenar, J.F., Peterson, M.L., Stephens, P.W., Zhang, Z., Zimenkov, Y. and Hickey, M.B. (2007) Celecoxib: Nicotinamide Dissociation Using Excipients to Capture the Cocrystal's Potential. Molecular Pharmacology, 4, 386-400.

https://doi.org/10.1021/mp0700108

[10] Berry, D.J., Seaton, C.C., Clegg, W., Harrington, R.W., Coles, S.J., Horton, P.N., et al. (2008) Applying Hot-Stage Microscopy to Co-Crystal Screening: A Study of Nicotinamide with Seven Active Pharmaceutical Ingredients. Crystal Growth \& Design, 8, 1697-1712. https://doi.org/10.1021/cg800035w

[11] Chieng, N., Hubert, M., Saville, D., Rades, T. and Aaltonen, J. (2009) Formation Kinetics and Stability of Carbamazepine-Nicotinamide Cocrystals Prepared by $\mathrm{Me}$ chanical Activation. Crystal Growth \& Design, 9, 2377-2386. https://doi.org/10.1021/cg801253f

[12] Porter, W., Elie, S. and Matzger, A. (2008) Polymorphism in Carbamazepine Cocrystals. Crystal Growth \& Design, 8, 14-16. https://doi.org/10.1021/cg701022e

[13] Cheney, M.L., Shan, N., Healey, E.R., Hanna, M., Wojtas, L., Zaworotko, M.J., et al. (2010) Effects of Crystal Form on Solubility and Pharmacokinetics: A Crystal Engineering Case Study of Lamotrigine. Crystal Growth \& Design, 10, 394-405. https://doi.org/10.1021/cg901010v

[14] Karimi-Jafari, M., Padrela, L., Walker, G.M. and Croker, D.M. (2018) Creating Cocrystals: A Review of Pharmaceutical Cocrystal Preparation Routes and Applications. Crystal Growth \& Design, 18, 6370-6387.

https://doi.org/10.1021/acs.cgd.8b00933

[15] Douroumis, D., Ross, S.A. and Nokhodchi, A. (2017) Advanced Methodologies for Cocrystal Synthesis. Advanced Drug Delivery Reviews, 117, 178-195. https://doi.org/10.1016/j.addr.2017.07.008 